\title{
Functional Recovery after Scutellarin Treatment in Transient Cerebral Ischemic Rats: A Pilot Study with ${ }^{18}$ F-Fluorodeoxyglucose MicroPET
}

\author{
Jin-hui Li, ${ }^{1,2,3,4,5}$ Jing Lu, ${ }^{6}$ and Hong Zhang ${ }^{1,3,4,5}$ \\ ${ }^{1}$ Department of Nuclear Medicine, Second Affiliated Hospital of Zhejiang University School of Medicine, Hangzhou, \\ Zhejiang 310009, China \\ ${ }^{2}$ Department of Traditional Chinese Medicine \& Rehabilitation, Second Affiliated Hospital of Zhejiang University School of Medicine, \\ Hangzhou, Zhejiang 310009, China \\ ${ }^{3}$ Zhejiang University Medical PET Center, Hangzhou, Zhejiang 310009, China \\ ${ }^{4}$ Institute of Nuclear Medicine and Molecular Imaging of Zhejiang University, Hangzhou, Zhejiang 310009, China \\ ${ }^{5}$ Key Laboratory of Medical Molecular Imaging of Zhejiang Province, Hangzhou, Zhejiang 310009, China \\ ${ }^{6}$ Department of Neurobiology, Zhejiang University School of Medicine, Hangzhou, Zhejiang 310058, China
}

Correspondence should be addressed to Hong Zhang; hzhang21@gmail.com

Received 28 February 2013; Accepted 12 April 2013

Academic Editor: Mei Tian

Copyright (C) 2013 Jin-hui Li et al. This is an open access article distributed under the Creative Commons Attribution License, which permits unrestricted use, distribution, and reproduction in any medium, provided the original work is properly cited.

Objective. To investigate neuroprotective effects of scutellarin $(\mathrm{Scu})$ in a rat model of cerebral ischemia with use of ${ }^{18} \mathrm{~F}$ fluorodeoxyglucose $\left({ }^{18} \mathrm{~F}-\mathrm{FDG}\right)$ micro positron emission tomography (microPET). Method. Middle cerebral artery occlusion was used to establish cerebral ischemia. Rats were divided into 5 groups: sham operation, cerebral ischemia-reperfusion untreated (CIRU) group, Scu-25 group (Scu $25 \mathrm{mg} / \mathrm{kg} / \mathrm{d}$ ), Scu-50 group (Scu $50 \mathrm{mg} / \mathrm{kg} / \mathrm{d}$ ), and nimodipine (10 mg/Kg/d). The treatment groups were given for 2 weeks. The therapeutic effects in terms of cerebral infarct volume, neurological deficit scores, and cerebral glucose metabolism were evaluated. Levels of vascular density factor (vWF), glial marker (GFAP), and mature neuronal marker $(\mathrm{NeuN})$ were assessed by immunohistochemistry. Results. The neurological deficit scores were significantly decreased in the Scu-50 group compared to the CIRU group $(P<0.001) .{ }^{18} \mathrm{~F}-\mathrm{FDG}$ accumulation in the ipsilateral cerebral infarction increased steadily over time in Scu-50 group compared with CIRU group $(P<0.01)$ and Scu-25 group $(P<0.01)$. Immunohistochemical analysis demonstrated Scu-50 enhanced neuronal maturation. Conclusion. ${ }^{18}$ F-FDG microPET imaging demonstrated metabolic recovery after Scu-50 treatment in the rat model of cerebral ischemia. The neuroprotective effects of Scu on cerebral ischemic injury might be associated with increased regional glucose activity and neuronal maturation.

\section{Introduction}

Positron emission tomography (PET) is widely used for the clinical evaluation of neurological disease. The ${ }^{18} \mathrm{~F}$ fluorodeoxy-D-glucose $\left({ }^{18} \mathrm{~F}-\mathrm{FDG}\right)$ method has been used for quantitative measurement of glucose utilization. In our previous study, we have measured the ischemic damage in the brain by means of ${ }^{18}$ F-FDG and demonstrated that a PET imaging scan might serve as a noninvasive therapeutic followup for individual animals [1]. We previously used alternative medicine therapies, including herbs and formulas, to examine cerebral ischemic reperfusion [2]. However, the increasing use of traditional therapies demands more scientific evidence for the principles behind them and for their effectiveness. Scutellarin (Scu), the main bioactive component of Erigeron breviscapus, has been used in the treatment of cerebral and heart vascular diseases $[3,4]$. Moreover, it also inhibits the replication of several strains of HIV-1 [5] and diminishes hyperglycemia [6]. A preliminary study revealed that neuroprotection by Scu is mediated by blocking the sodium current of hippocampal neurons [7], by inhibiting the formation of hydrogen peroxide and the 
neuroinflammatory reaction of microglia [8] and apoptosisinducing pathways [9], and by increasing constitutive nitric oxide synthase (cNOS) [10]. Many brain injuries, such as cerebral ischemia [11-13], hypoxia [14], and hemorrhage [15], are associated with the proliferation of endogenous neural progenitor cells. The exact function of these cells and their fate following cerebral ischemic reperfusion is still not clear [16]. Therefore, we aimed to monitor neural progenitor cells for a longer period after ischemic stroke in adult rat brain $[11,17,18]$. However, the recovery of neurological function mainly depends on the neurovascular unit (NVU), that is, on interactions between astrocytes, brain microvessels, and their environment [19], all of which may exert either protective or harmful actions.

NVU as a paradigm $[20,21]$ is broadly applicable to cerebral ischemia. The basement membrane that underlies the endothelial cells is a key structure for maintaining the integrity of the neurovascular unit, and a free radical scavenger can be a viable agent for inhibiting tissue plasminogen activator [22]. Glial scarring is commonly thought to have an adverse effect after stroke. However, a new study now suggests that reactive astrocytes in the peri-infarct cortex may additionally contribute to neurovascular remodeling [23]. Stroke-generated new neurons and neuroblasts that were probably already present before the insult migrated into the severely damaged area of the cortex, where they express markers of developing, mature, and striatal-like spiny neurons [24]. We tested the hypothesis that cerebral ischemic damage prolongs the impairment of the metabolic coupling of neurovascular units and neural cells. Impaired neurovascular coupling was explained by reduced vascular reactivity and suppressed function of cortical inhibitory interneurons. Intriguingly, some flavonoids such as galangin, chrysin, and pinocembrin have the capacity to shield the neurovascular unit in rats [25] and ensure neuroprotection against cerebral ischemia/reperfusion (I/R) injury [20]. Moreover, we speculate that neurovascular plasticity is characterized by increasing regional glucose activity and neuron numbers that provide protection against stroke weeks longer than previously established.

To better understand the mechanisms involved and to be able to make proposals for the prevention of cerebral I/R injury, as well as give a prognosis, further study is required. Scu might have bidirectional regulation effects, as well as being able to dispose of paradoxical diseases, like hemorrhagic cerebral infarction simultaneously $[26,27]$. Therefore, the present study mainly explored the effects of Scu on neuronal regeneration, astrocyte activity, and microvascular and regional glucose activity in transient middle-cerebral artery-occlusion- (MCAO-) induced focal cerebral ischemia in rats.

\section{Materials and Methods}

2.1. Animals, Experimental Groups, and Outline of the Experiment. Adult male SD rats weigh 250-280 g with the lightdark cycle each 12 hours, filtrate water, and total nutrient feed. Then, all rats were divided into five experimental groups (12 rats for each group): sham group, a cerebral ischemia-reperfusion untreated (CIRU) group, Scu-25 group (Scu $25 \mathrm{mg} / \mathrm{Kg} / \mathrm{d}$ ), Scu-50 group (Scu $50 \mathrm{mg} / \mathrm{Kg} / \mathrm{d}$ ), and nimodipine group $(10 \mathrm{mg} / \mathrm{Kg} / \mathrm{d})$. All the groups were fed by oral gavage feeding for a period of 2 weeks. Behavioral tests and PET imaging were performed at 1, 7, 14, 21, and 28 days after MCAO. All rats were euthanized at the 28 days after MCAO for immunohistochemical detection.

\subsection{Animal Model of Middle Cerebral Artery Occlusion} (MCAO). All rats were anesthetized by injections of $1.5 \%$ pentobarbital sodium $(50 \mathrm{mg} / \mathrm{kg}$, intraperitoneal). Focal brain ischemia was induced by the intraluminal suture. A midline skin incision in the neck was followed by subsequent exploration of the right common carotid artery (CCA), the external carotid artery (ECA), and the internal carotid artery (ICA). A 4-0 monofilament nylon suture with a rounded tip was introduced into the left internal carotid by arteriotomy and advanced $18-20 \mathrm{~mm}$ past the carotid bifurcation. After $1 \mathrm{~h}$ of $\mathrm{MCAO}$, reperfusion was achieved by withdrawing the endovascular suture to the stump of the ECA, and the skin was then sutured [28]. Rectal temperature was monitored continuously and maintained at $36.5-37.5^{\circ} \mathrm{C}$ for the duration of the surgery. The rats were housed in environmentally enriched conditions under a $12 \mathrm{~h}$ light $/ 12 \mathrm{~h}$ dark cycle throughout the experiments and were allowed free access to food and water.

2.3. Neurological Functional Tests. A modified neurological severity score (mNSS) test was performed [29], post-MCAO at $24 \mathrm{~h}$ and at 1,2, 3, and 4 weeks after reperfusion. The mNSS was graded on a scale of 0 to 18 (normal score, 0 ; maximal deficit score, 18). In the severity scores of injury, 1 score point is awarded for the inability to perform the test or for the lack of a tested reflex; thus, the higher the score, the more severe the injury.

2.4. Triphenyltetrazolium Chloride Assessment. Total 15 rats (each group, $n=5$ ) were anesthetized and sacrificed by rapid decapitation. The rat brain was collected at $7 \mathrm{~d}$ after reperfusion. The brain was kept at $-20^{\circ} \mathrm{C}$, then sliced into $2 \mathrm{~mm}$ thick coronal sections and stained with $1 \%$ 2,3,5triphenyltetrazolium chloride (Sigma Chemicals) solution for $15 \mathrm{~min}$ at $37^{\circ} \mathrm{C}$, and fixed in $4 \%$ paraformaldehyde at $4^{\circ} \mathrm{C}$ overnight. After fixation, the brain slices were scanned using a flat-bed scanner. Infarct volume was quantified with professional image analysis software (Analysis Life Science Professional). Infarct volumes were calculated by adding up the infarct areas in six brain slices by thickness $(2 \mathrm{~mm})$ slightly.

2.5. MicroPET Imaging and Data Analysis. At 24 hours after reperfusion and at 1, 2, 3, and 4 weeks after MCAO, rats were anesthetized with isofluorane (2\%) and injected with approximately $18.5 \mathrm{MBq}$ of ${ }^{18} \mathrm{~F}-\mathrm{FDG}$ via the tail vein. At $30 \mathrm{~min}$ after ${ }^{18} \mathrm{~F}-\mathrm{FDG}$ injection, rats were anesthetized with isofluorane $(2 \%)$, and a $10 \mathrm{~min}$ static acquisition was 
TABLE 1: Values of five groups of neurological deficit after cerebral ischemia/reperfusion in different days (mean \pm SEM).

\begin{tabular}{|c|c|c|c|c|c|c|}
\hline \multirow{2}{*}{ Groups } & \multirow{2}{*}{$n$} & \multicolumn{5}{|c|}{ Neurologic score } \\
\hline & & Day 0 & Day 7 & Day 14 & Day 21 & Day 28 \\
\hline Sham & 6 & $0 \pm 0$ & $0 \pm 0$ & $0 \pm 0$ & $0 \pm 0$ & $0 \pm 0$ \\
\hline CIRU & 6 & $12.00^{*} \pm 0.58$ & $11.33^{*} \pm 0.33$ & $10.33^{*} \pm 0.33$ & $9.33^{*} \pm 0.33$ & $7.67^{*} \pm 0.67$ \\
\hline Scu-50 & 6 & $12.67^{*} \pm 0.88$ & $7.67^{* * *} \pm 0.33$ & $7.67^{* * *} \pm 0.33$ & $6.33^{* * *} \pm 0.33$ & $2.67^{* * *} \pm 0.33$ \\
\hline Scu-25 & 6 & $12.33^{*} \pm 0.33$ & $9.67^{* *} \pm 0.33$ & $8.67^{* *} \pm 0.33$ & $7.33^{* * *} \pm 0.33$ & $5.67^{* *} \pm 0.33$ \\
\hline Nimodipine & 6 & $11.67^{*} \pm 0.33$ & $10.33 \pm 0.33$ & $9.33 \pm 0.33$ & $7.67^{* *} \pm 0.33$ & $5.67^{* *} \pm 0.33$ \\
\hline
\end{tabular}

Values are mean \pm SD.

${ }^{*} P<0.01$; significantly higher compared with values of sham group.

${ }^{* *} P<0.05$; significantly lower compared with values of CIRU group.

${ }^{* * *} P<0.01$; significantly lower compared with values of CIRU group.

performed with a midhead machine, and images were reconstructed using a maximum a posteriori algorithm. Uptake was calculated as the percentage of injected dose per gram $(\% \mathrm{ID} / \mathrm{g})$ of tissue using the ASIPro 6.0.5.0 software (CTI Concorde Microsystems, LLC profile in Knoxville, TN, USA) at the Medical PET Center of Zhejiang University. Then, rats were anesthetized with isoflurane and prone positioned on the microPET R4 rodent model scanning gantry (Siemens Preclinical Solutions, Knoxville, TN, USA). The scanner has a computer-controlled bed and $10.8 \mathrm{~cm}$ transaxial and $8 \mathrm{~cm}$ axial fields of view. The voxel size was $0.845 \mathrm{~mm}$ on a side, and the full width at half maximum was $1.66,1.65$, and $1.84 \mathrm{~mm}$ for tangential, radial, and axial orientations, respectively. In each scan, 3 different regions of interest (ROI), $0.5 \mathrm{~mm}$ in diameter manually drawn by creating a volume of interest in the central of the ischemic area and using the mean dose per gram of tissue (\%ID/g), were averaged. The lesion-tonormal homologous cerebellum ratio $(\mathrm{L} / \mathrm{N})$ was used for semiquantitative analysis. $\mathrm{L} / \mathrm{N}$ ratio was calculated using the following formula: $\mathrm{L} / \mathrm{N}$ ratio $=$ mean counts per pixel of lesion ROIs/mean counts per pixel of normal homologous cerebellum ROIs.

2.6. Immunohistochemical Assessment. Animals were overdosed with $10 \%$ chloral hydrate, after which their thoracic cavities were opened and perfused intracardially with normal saline followed by $4 \%$ paraformaldehyde in $0.1 \mathrm{M}$ phosphate buffer ( $\mathrm{pH}$ 7.4). The brain was removed and fixed by immersion in fresh fixative overnight and stored in $30 \%(\mathrm{v} / \mathrm{v})$ phosphate-buffered sucrose overnight. Differentiation of the nature of cells was determined by colocalizing markers for different cell types (vWF: rabbit polyclonal antibody, DAKO A008229,1:200; GFAP: rabbit polyclonal antibody, Abcam ab7260,1:300; NeuN: mouse monoclonal antibody, clone: A60, Millipore MAB377, 1:200). Sections were blocked and incubated overnight at $4^{\circ} \mathrm{C}$ with primary antibodies. After having been washed in PBS, sections were incubated with conjugated secondary antibodies (antimouse or antirabbit Alexa 568) for $1 \mathrm{~h}$ at $37^{\circ} \mathrm{C}$ following 2-3 min rinsing in PBS with diaminobenzidine chromagen $(0.5 \mathrm{mg} / \mathrm{mL})$, rinsed twice in distilled water, $50 \mathrm{~mL}$ hematoxylin stained for 2$5 \mathrm{~min}$, differentiated with $1 \%$ hydrochloric acid alcohol, and dehydrated with dimethylbenzene transparent, blue-based cover slipped with glycerin (Shanghai Sengene biotech Co.,
Ltd.), after which the sections were examined under a light microscope and IODs of vWF, GFAP, and NeuN quantitative analysis by using Image-Pro Plus 5.0 software (Media Cybernetics).

2.7. Statistical Analysis. All results were expressed as mean \pm SEM accompanied by the number of observations. Continuous variables were compared by the unpaired Students $t$-test, and multiple groups were compared by ANOVA using the Bonferroni correction. Significant differences were accepted when $P<0.05$.

\section{Results}

3.1. Effects on Neurological Deficit. After MCAO, the neurological deficit score of the CIRU group was significantly higher than that of the sham group $(P<0.01)$ on days 0,7 , 14,21 , and 28, whereas those of the Scu-50 group (50 mg/kg/d) and Scu-25 group ( $25 \mathrm{mg} / \mathrm{kg} / \mathrm{d})$ were lower than those of the occluded group on day 7 and day $14(P<0.001, P<0.05$ separately). The scores of the Scu-50 group $(P<0.001)$, Scu25 group $(P<0.01)$, and nimodipine group $(P<0.05)$ were significantly lower than that of the model group on day 21 . And the scores of the Scu-50 group $(P<0.001)$, Scu-25 group $(P<0.05)$, and nimodipine group $(P<0.05)$ were lower than that of the occluded group on day 28. Furthermore, the scores of the Scu-50 group were lower than those of the Scu25 group and nimodipine group $(P<0.01)$ (Table 1 and Figure 1).

3.2. Triphenyltetrazolium Chloride Evaluation. The infarct volume in the occluded group was significantly increased compared to the sham group $(P<0.001)$. The Scu-50 group showed the smallest infarct volume $\left(56.0 \pm 2.00 \mathrm{~mm}^{3}\right.$, $P<0.001)$, followed by the Scu-25 group $\left(74.3 \pm 3.51 \mathrm{~mm}^{3}\right.$, $P<0.001)$ and the nimodipine group $\left(98.3 \pm 2.06 \mathrm{~mm}^{3}\right.$, $P<0.001)$, when compared with the CIRU group $(244.3 \pm$ $7.76 \mathrm{~mm}^{3}$ ) (Figure 2 and Table 2).

3.3. Effects on Brain Glucose Metabolism. Glucose metabolism in the brain was significantly reduced in the damaged region of the CIRU group compared with the sham group $(P<0.01)$. Glucose metabolism of Scu-50 group was 


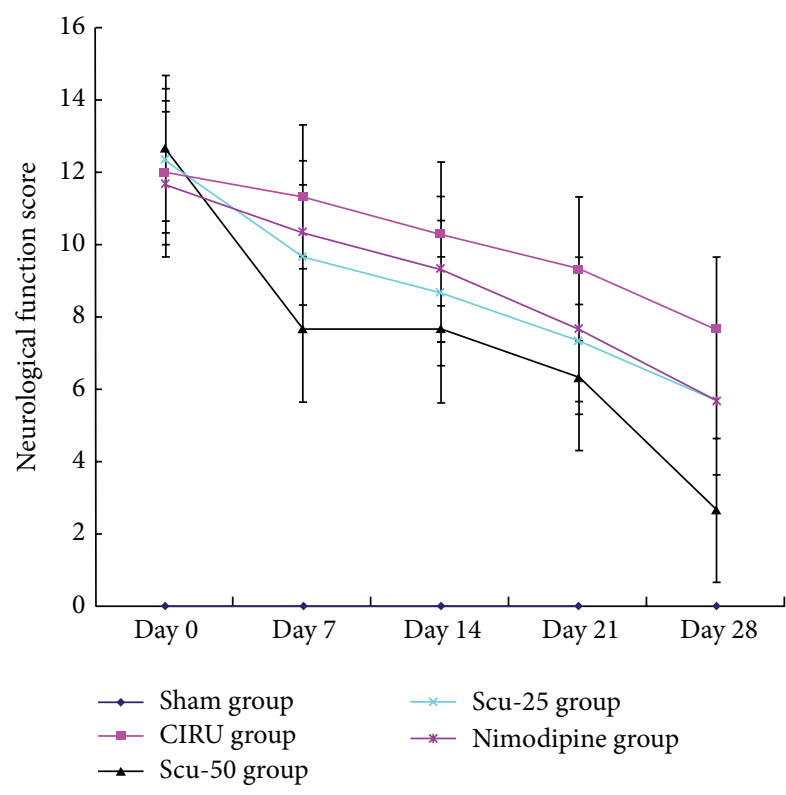

FIGURE 1: Neurological functional tests to describe rat neurological scores of middle cerebral artery occlusion-induced focal cerebral ischemia. Sham group (sham), cerebral ischemia-reperfusion untreated group (CIRU), scutellarin $50 \mathrm{mg} / \mathrm{Kg}$ group (Scu-50), scutellarin $25 \mathrm{mg} / \mathrm{Kg}$ group (Scu-25), and nimodipine $10 \mathrm{mg} / \mathrm{Kg}$ group (Nimodipine) in days $0,7,14,21$, and 28 , respectively.

increased significantly $(P<0.01)$ on day 14 compared with the CIRU group, whereas there was no significance of the Scu-25 group and the nimodipine group in the right cerebral ischemic regions compared with the CIRU group on days 7, 14, 21, and 28. Intriguingly, ${ }^{18}$ F-FDG uptake of focal ischemic lesion in Scu-50 was also higher than Scu-25 group (Table 3). Furthermore, the Scu-50 group yielded the best glucose metabolism increasing compared with CIRU group, Scu-25 group, and nimodipine treatment groups (Figure 3).

3.4. Expression of Vascular Density Factor (vWF), GFAP, and NeuN Immunoreactivity. Immunohistochemical studies were performed to determine whether Scu treatment can induce neurogenesis and angiogenesis. NeuN was used as a mature neuronal marker, GFAP as the mature astrocytes marker, and vWF as the endothelial cell marker. We counted the number of cells immunostaining positively the NeuN or vWF and calculated the mean values and SEMs. All the data of immunochemistry analysis were summarized in Figure 4 and Table 4. For the vWF expression, immunoreactivity was higher in the CIRU group and treated groups than in the sham group $(P<0.05)$. GFAP-positive cell number was significantly increased in the $50 \mathrm{mg} / \mathrm{Kg}$ Scu and $25 \mathrm{mg} / \mathrm{Kg} \mathrm{Scu}$ groups compared with the sham group $(P<0.05)$. However, all the Scu groups showed no significance compared with the CIRU group. Expression of NeuN intensity in the Scu50 group significantly increased compared with the CIRU group $(P<0.001)$. NeuN intensity in the Scu-50 group also increased significantly compared with the nimodipine group $(P<0.05)$. Interestingly, the Scu-50 group showed
TABLE 2: Infarct volume of cerebral ischemia after cerebral IR with TTC stain at the day $14\left(\right.$ mean \pm SEM, $\left.\mathrm{mm}^{3}\right)$.

\begin{tabular}{llc}
\hline Group & $n$ & Infarct volume \\
\hline Sham & 3 & $0.0 \pm 0.00$ \\
CIRU & 3 & $244.3^{*} \pm 7.76$ \\
Scu-50 & 3 & $56.0^{* *} \pm 2.00$ \\
Scu-25 & 3 & $74.3^{* *, \mathrm{w}} \pm 3.51$ \\
Nimodipine & 3 & $98.3^{* *} \pm 3.06$ \\
\hline
\end{tabular}

Values are mean \pm SD.

${ }^{*} P<0.01$; significantly higher compared with values of sham group.

${ }^{* *} P<0.01$; significantly lower compared with values of CIRU group.

${ }^{\mathrm{w}} \mathrm{P}<0.01$; significantly lower compared with values of Scu- 25 group.

a significant higher expression of NeuN immunoreactivity compared with the Scu-25 group $(P<0.05)$.

\section{Discussion}

Following cerebral ischemia, the extracellular concentration of excitatory amino acids increases, resulting in the excitatory cell death in ischemic neuronal damage [30]. Although sequential metabolic changes in permanent cerebral ischemia have been reported [31], the effect of reperfusion in local cerebral ischemia on glucose metabolism is less clear. In order to study the time course of the changes in glucose metabolism following middle cerebral artery occlusion-reperfusion model and the effects of Scu on glucose metabolism, the ${ }^{14} \mathrm{C}$-deoxyglucose method was used [32]. Hypermetabolism occurred at 30 minutes after the middle cerebral artery (MCA) occlusion and reached a peak at $60 \mathrm{~min}$ after ischemia in both ischemic core and penumbra regions [33].

Breviscapine, one plant flavonoids found from Erigeron breviscapus, could dilate brain blood vessels, increased cerebral blood flow and cardiac coronary flow, reduce blood viscosity, and improve microcirculation [34]. Traditionally it has been used for thousand years. We estimated that the main ingredient of Erigeron breviscapus, Scu, might inhibite both increased glucose metabolism during ischemia and decreased glucose metabolism during reperfusion. These findings support the hypothesis that excitation-induced hypermetabolism plays a major role in the ischemic insult following focal cerebral vascular occlusion $[35,36]$.

$\mathrm{Scu}$, one of the ingredients of breviscapine, was used for neuronal damage. This compound was tested for increasing $\left[\mathrm{Ca}^{2+}\right]$ ion and activating protein kinase $\mathrm{C} \gamma(\mathrm{PKC} \gamma)$ following cerebral ischemia and reperfusion [37] and was also found to play a vital role in protecting PC12 cells from cobalt chloride-induced apoptosis by scavenging reactive oxygen species, inhibiting p38 phosphorylation, upregulating Bcl-XL expression, and decreasing caspase-3 activity [38]. Moreover, Scu attenuated $\mathrm{H}_{2} \mathrm{O}_{2}$-induced cytotoxicity, lipid peroxidation, and loss of DNA [39] and inhibited hydrogen peroxide increased activity of cNOS and oxidative damage induced by superoxide in synaptosomes [40]. Also, it showed an inhibition of polyadenosine diphosphate ribose 


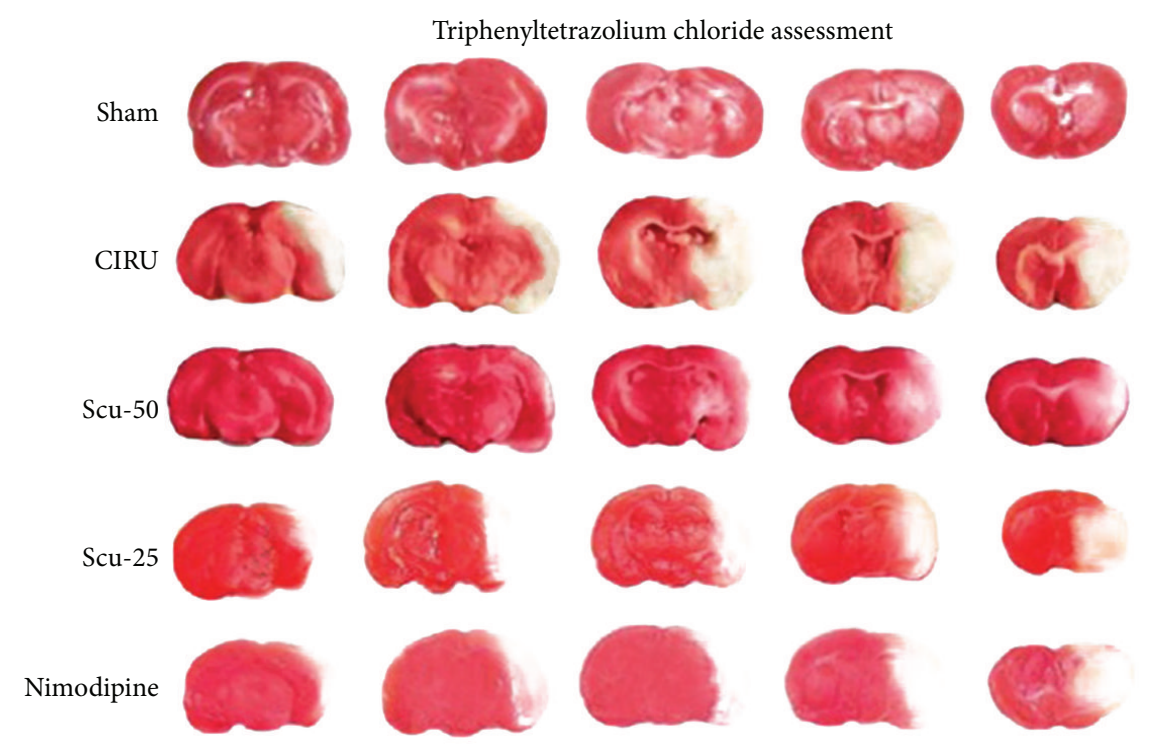

FIGURE 2: 2,3,5-Triphenyltetrazolium chloride (TTC) staining to delineate rat brain infarcts of middle cerebral artery occlusion-induced focal cerebral ischemia. Sham group (sham), cerebral ischemia-reperfusion untreated group (CIRU), scutellarin $50 \mathrm{mg} / \mathrm{Kg}$ group (Scu-50), scutellarin $25 \mathrm{mg} / \mathrm{Kg}$ group (Scu-25), and nimodipine $10 \mathrm{mg} / \mathrm{Kg}$ group (nimodipine) at day 14 after reperfusion.

TABLE 3: Effects on glucose metabolism after cerebral IR in different days (mean \pm SEM, ratio of lesion to cerebellum).

\begin{tabular}{lcccccc}
\hline \multirow{2}{*}{ Groups } & $n$ & \multicolumn{4}{c}{ Glucose metabolism of rat brain using ${ }^{18}$ F-FDG microPET } \\
& & Day 0 & Day 7 & Day 14 & Day 21 & Day 28 \\
\hline Sham & 6 & $0.96 \pm 0.02$ & $0.91 \pm 0.03$ & $0.85 \pm 0.03$ & $0.99 \pm 0.03$ & $1.08 \pm 0.03$ \\
CIRU & 6 & $0.76^{*} \pm 0.03$ & $0.67^{*} \pm 0.03$ & $0.65^{*} \pm 0.03$ & $0.60^{*} \pm 0.04$ & $0.62^{*} \pm 0.04$ \\
Scu-50 & 6 & $0.75^{* \mathrm{w}} \pm 0.02$ & $0.71^{* \mathrm{w}} \pm 0.04$ & $0.74^{* *} \pm 0.01$ & $0.75^{* *} \pm 0.04$ & $0.76^{* *} \pm 0.03$ \\
Scu-25 & 6 & $0.54^{*} \pm 0.03$ & $0.49^{*} \pm 0.05$ & $0.45^{*} \pm 0.02$ & $0.46^{*} \pm 0.04$ & $0.41^{*} \pm 0.04$ \\
Nimodipine & 6 & $0.63^{*} \pm 0.03$ & $0.44^{*} \pm 0.03$ & $0.43^{*} \pm 0.05$ & $0.40^{*} \pm 0.03$ & $0.51^{*} \pm 0.02$ \\
\hline
\end{tabular}

Values are mean \pm SD.

${ }^{*} P<0.01$; significantly higher compared with values of sham group.

${ }^{* *} P<0.01$; significantly higher compared with values of CIRU group.

${ }^{\mathrm{w}} P>0.05$; compared with values of CIRU group.

polymerase-dependent mitochondrial dysfunction and subsequent translocation of apoptosis-inducing factor in vitro [41]. In this study, compared with the control group, Scu $(50 \mathrm{mg} / \mathrm{kg})$ reduced neurons apoptosis in cerebral infarction rats, and a dose-dependent Scu influence degree of the effects on anticerebral ischemia was observed in vivo [42].

Our data confirmed that the dose-dependent increased brain glucose metabolism is as observed earlier in brain PET studies, since the cerebellum does not show alterations under the circumstances $[43,44]$ and the ratio of lesion to cerebellum region index is more commonly used in PET studies $[36,45]$. During the delayed phase, brain angiogenesis may provide the critical neurovascular substrates for neuronal renew. As we know, ${ }^{18}$ F-FDG uptake and regional cerebral blood flow represent neuron activity both in vitro and in vivo [46]. Although the ratio of ischemic cortex to nonischemic cortex has no consistency to the actual effect, it is not influenced by the weight of the rat or the amount of radionuclide flow at the injection points. So we chose the ratio of ischemic cortex to nonischemic cerebellum which is more stable than the ratio of the lesion/contralateral cortex region of brain glucose metabolism. One result showed that glucose metabolism utilization in cerebellum decreased at all times in special neurons of focal ischemia rat, which was not totally consistent with our results [47].

Scu, also called chelerythrine chloride, was considered the PKC inhibitors, which have potential as anti-ischemia agents in vitro $(\mathrm{IC} 50=0.66 \mu \mathrm{M})$ and in vivo $[48,49]$. And as we know PKC $\gamma$ has the most abundant distribution in the cerebellum, hippocampus, and cerebral cortex [50]. We speculated that Scu primarily and specially blocks ischemic tissues in rat brain by activating PKC $\gamma$ and exerting protective effects against cerebral ischemia-reperfusion injury in vivo.

Because the expression of vWF activity was higher in the CIRU group and treatment groups than in the sham group, we presume to focal ischemia, the cerebral microvasculature multiple dynamic responses evolved through microvascular propagation of ischemic and peri-ischemic regions and astrocyte nourishment in treatment groups [51]. In the NVU, on one hand, astrocytes protect neurons but are a danger 


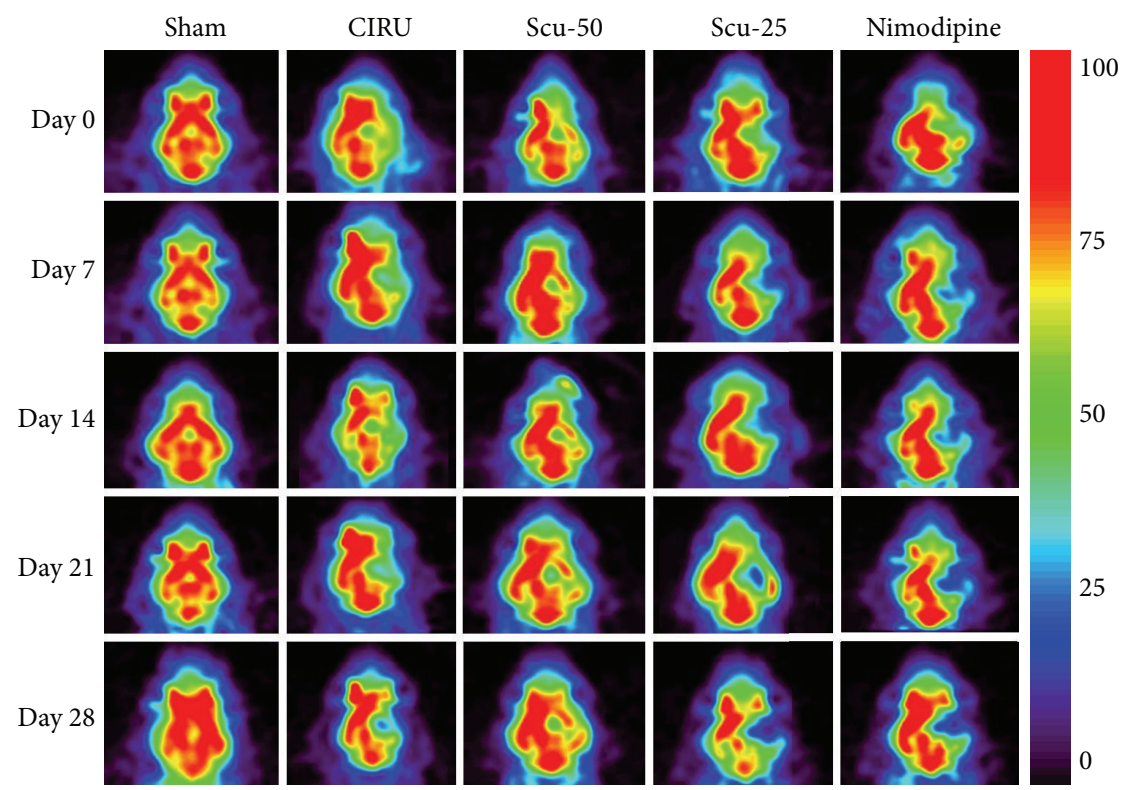

(a)

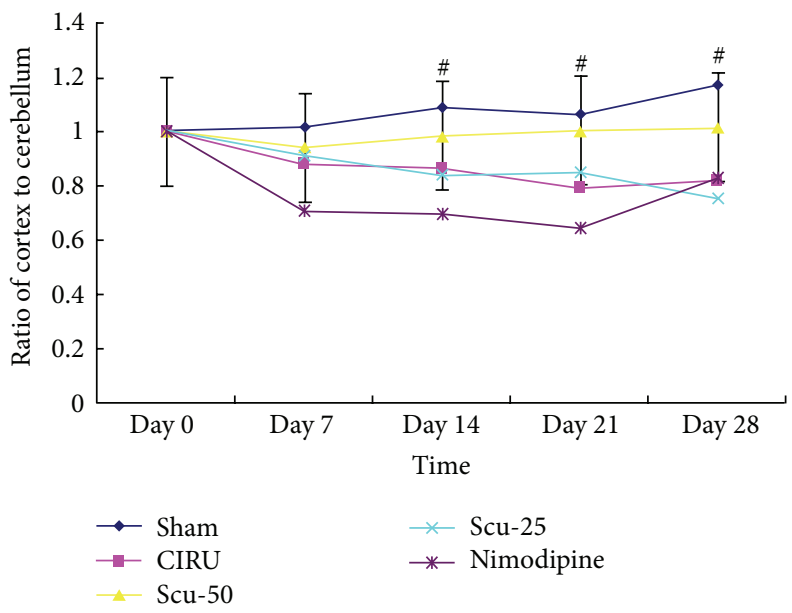

(b)

FIgURE 3: (a) ${ }^{18}$ FDG-PET images of activity of cerebral ischemic regions of the glucose metabolism. Sham group (sham), cerebral ischemiareperfusion untreated group (CIRU), scutellarin $50 \mathrm{mg} / \mathrm{Kg}$ group ( $\mathrm{Scu}-50$ ), scutellarin $25 \mathrm{mg} / \mathrm{Kg}$ group (Scu-25), and nimodipine $10 \mathrm{mg} / \mathrm{Kg}$ group (nimodipine) in days $0,7,14,21$, and 28 respectively. (b) Ratios of cortex to cerebellum in different regions in the brain of the glucose metabolism sham group (sham), cerebral ischemia-reperfusion untreated group (CIRU), scutellarin $50 \mathrm{mg} / \mathrm{Kg}$ group (Scu-50), scutellarin $25 \mathrm{mg} / \mathrm{Kg}$ group (Scu-25), and nimodipine $10 \mathrm{mg} / \mathrm{Kg}$ group (nimodipine) in days $0,7,14,21$, and 28, respectively.

TABLE 4: The expression of NVU immunoreactive (mean \pm SEM, numbers of positive cells/field of vision).

\begin{tabular}{lcccr}
\hline Group & $n$ & vWF & GFAP & NeuN \\
\hline Sham group & 3 & $7.33 \pm 2.51$ & $2222.24 \pm 481.44$ & $46.67 \pm 8.74$ \\
CIRU group & 3 & $11.33^{*} \pm 2.52$ & $2936.06 \pm 703.33$ & $47.67 \pm 8.14$ \\
Scu-50 group & 3 & $13.0^{*} \pm 1.00$ & $3438.27^{*} \pm 805.82$ & $75.00^{* *, \mathrm{w}, * * *} \pm 6.08$ \\
Scu-25 group & 3 & $10.0^{*} \pm 1.00$ & $3509.09^{*} \pm 281.83$ & $48.33 \pm 5.51$ \\
Nimodipine group & 3 & $14.0^{*} \pm 1.73$ & $3016.93 \pm 113.45$ & $59.67 \pm 8.33$ \\
\hline
\end{tabular}

Values are means \pm SD.

${ }^{*} P<0.05$; significantly lower compared with values of sham group.

${ }^{* *} P<0.01$; significantly higher compared with values of Scu- 25 group.

${ }^{* * *} P<0.001$; significantly higher compared with values of CIRU group.

${ }^{\mathrm{w}} P<0.05$; significantly higher compared with values of nimodipine group. 


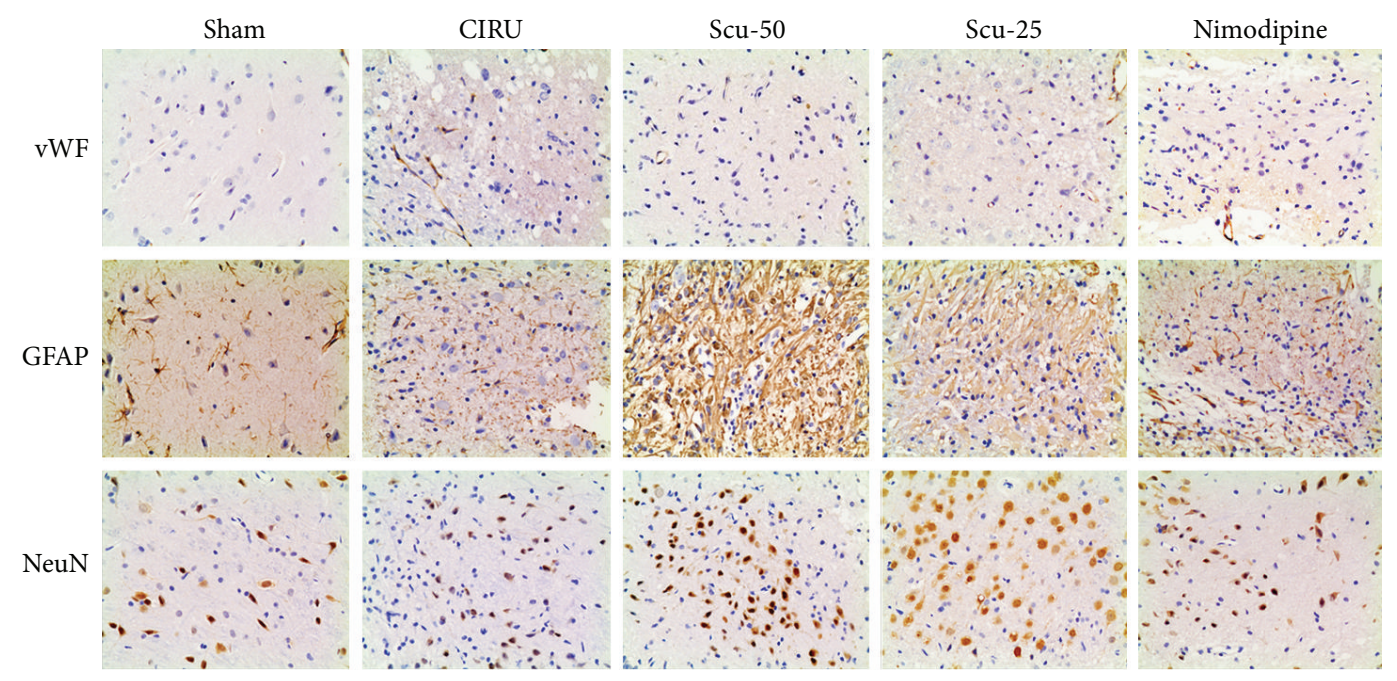

Figure 4: Vascular density factor (vWF), mature glial marker (GFAP), and neurogenesis markers (NeuN) immunostained tissue (magnification 200x) of middle cerebral artery occlusion-induced focal cerebral ischemia in rats. Sham group (sham), model group (CIRU), scutellarin $50 \mathrm{mg} / \mathrm{Kg}$ group (Scu-50), scutellarin $25 \mathrm{mg} / \mathrm{Kg}$ group (Scu-25), and nimodipine group (nimodipine) at day 28 after reperfusion.

to them. Our study provides evidence that Scu enhances neuronal survival and may improve special neurons glucose metabolism of cerebral ischemia [52, 53]. Moreover, Scu increased ${ }^{18} \mathrm{~F}$-FDG uptake of neurons might be through facilitative expressing of GFAP-positive cells [54]. On the other hand, focal cerebral ischemia preferentially affects neurons distant from their neighboring microvessels [55]. In this study, the number of astrocytic GFAP-positive cells increased in all treatment groups and CIRU group compared to the sham group. Furthermore, $50 \mathrm{mg} / \mathrm{kg}$ of Scu not only increased glucose metabolism of neurons at the early stage of cerebral damage but also increased expression of NeuN induced by cerebral ischemia. Scu-50 may have potential to the prevention and treatment of stroke compared with Scu-25 and Scu which have shown anti-ischemia reperfusion activity in a dose-dependent manner.

Nimodipine, a calcium channel blocker, originally developed for use in cardiovascular disorders. Our data indicated that nimodipine is recognized as an effective drug against cerebral ischemia but minor, when we set nimodipine previously as a positive control group, and therefore, we speculated that Scu might have potential protective effect by inhibiting influx of calcium ion against cerebral ischemia.

\section{Conclusion}

This study demonstrated the metabolic recovery after highdose Scu treatment by ${ }^{18} \mathrm{~F}$-FDG microPET imaging and the neuroprotective effects of Scu in a rat model of cerebral ischemia.

\section{Acknowledgments}

This work was partly sponsored by grants from the Natural Science Foundation of Zhejiang Province of China (Z2110230), Health Bureau of Zhejiang Province
(2010ZA075, 2011ZDA013), Science and Technology Bureau of Zhejiang Province (2012R10040), National Science Foundation of China (NSFC) (nos. 81202947, 81101023, and 81173468), and Ministry of Science and Technology of China (2011CB504400).

\section{References}

[1] H. Wan, H. Zhu, M. Tian et al., "Protective effect of chuanxiongzine-puerarin in a rat model of transient middle cerebral artery occlusion-induced focal cerebral ischemia," Nuclear Medicine Communications, vol. 29, no. 12, pp. 1113-1122, 2008.

[2] J. Yang, J. Li, J. Lu et al., "Synergistic protective effect of astragaloside IV-tetramethylpyrazine against cerebral ischemicreperfusion injury induced by transient focal ischemia," Journal of Ethnopharmacology, vol. 140, no. 1, pp. 64-72, 2012.

[3] L. L. Guo, Z. Z. Guan, and Y. L. Wang, "Scutellarin protects against Abeta-induced learning and memory deficits in rats: involvement of nicotinic acetylcholine receptors and cholinesterase," Acta Pharmacologica Sinica, vol. 32, no. 12, pp. 1446-1453, 2011.

[4] L. L. Lin, A. J. Liu, J. G. Liu, X. H. Yu, L. P. Qin, and D. F. Su, "Protective effects of scutellarin and breviscapine on brain and heart ischemia in rats," Journal of Cardiovascular Pharmacology, vol. 50, no. 3, pp. 327-332, 2007.

[5] G. H. Zhang, Q. Wang, J. J. Chen, X. M. Zhang, S. C. Tam, and Y. T. Zheng, "The anti-HIV-1 effect of scutellarin," Biochemical and Biophysical Research Communications, vol. 334, no. 3, pp. 812-816, 2005.

[6] Y. Su, W. Liu, L. Ma et al., "Scutellarin inhibits translocation of protein kinase $\mathrm{C}$ in diabetic thoracic aorta of the rat," Clinical and Experimental Pharmacology \& Physiology, vol. 39, no. 2, pp. 136-140, 2012.

[7] G. Zhang, S. Qiu, and H. Wei, "Scutellarin blocks sodium current in freshly isolated mouse hippocampal CA1 neurons," Neurochemical Research, vol. 36, no. 6, pp. 947-954, 2011.

[8] S. Wang, H. Wang, H. Guo, L. Kang, X. Gao, and L. Hu, "Neuroprotection of Scutellarin is mediated by inhibition of 
microglial inflammatory activation," Neuroscience, vol. 185, pp. 150-160, 2011.

[9] H. F. Zhang, X. M. Hu, L. X. Wang, S. Q. Xu, and F. D. Zeng, "Protective effects of scutellarin against cerebral ischemia in rats: evidence for inhibition of the apoptosis-inducing factor pathway," Planta Medica, vol. 75, no. 2, pp. 121-126, 2009.

[10] H. Liu, X. Yang, R. Tang, J. Liu, and H. Xu, "Effect of scutellarin on nitric oxide production in early stages of neuron damage induced by hydrogen peroxide," Pharmacological Research, vol. 51, no. 3, pp. 205-210, 2005.

[11] B. Li, C. S. Piao, X. Y. Liu et al., "Brain self-protection: the role of endogenous neural progenitor cells in adult brain after cerebral cortical ischemia," Brain Research, vol. 1327, pp. 91-102, 2010.

[12] N. Nakagomi, T. Nakagomi, S. Kubo et al., "Endothelial cells support survival, proliferation, and neuronal differentiation of transplanted adult ischemia-induced neural stem/progenitor cells after cerebral infarction," Stem Cells, vol. 27, no. 9, pp. 21852195, 2009.

[13] L. Li, Q. Jiang, G. Ding et al., "Effects of administration route on migration and distribution of neural progenitor cells transplanted into rats with focal cerebral ischemia, an MRI study," Journal of Cerebral Blood Flow and Metabolism, vol. 30, no. 3, pp. 653-662, 2010.

[14] L. Zhao, Q. Jiao, X. Chen et al., "mGluR5 is involved in proliferation of rat neural progenitor cells exposed to hypoxia with activation of mitogen-activated protein kinase signaling pathway," Journal of Neuroscience Research, vol. 90, no. 2, pp. 447-460, 2012.

[15] X. Xu, J. Zhang, X. Chen et al., "The increased expression of metabotropic glutamate receptor 5 in subventricular zone neural progenitor cells and enhanced neurogenesis in a rat model of intracerebral hemorrhage," Neuroscience, vol. 202, pp. 474-483, 2012.

[16] C. C. Shen, Y. C. Yang, M. T. Chiao, W. Y. Cheng, Y. S. Tsuei, and J. L. Ko, "Characterization of endogenous neural progenitor cells after experimental ischemic stroke," Current Neurovascular Research, vol. 7, no. 1, pp. 6-14, 2010.

[17] J. Minnerup, J. B. Kim, A. Schmidt et al., "Effects of neural progenitor cells on sensorimotor recovery and endogenous repair mechanisms after photothrombotic stroke," Stroke, vol. 42, no. 6, pp. 1757-1763, 2011.

[18] A. M. Osman, M. J. Porritt, M. Nilsson, and H. G. Kuhn, "Long-term stimulation of neural progenitor cell migration after cortical ischemia in mice," Stroke, vol. 42, no. 12, pp. 35593565, 2011.

[19] Y. Gürsoy-Özdemir, A. Can, and T. Dalkara, "Reperfusioninduced oxidative/nitrativie injury to neurovascular unit after focal cerebral ischemia," Stroke, vol. 35, no. 6, pp. 1449-1453, 2004.

[20] Y. H. Ran and H. Wang, "Iptakalim, an ATP-sensitive potassium channel opener, confers neuroprotection against cerebral ischemia/reperfusion injury in rats by protecting neurovascular unit cells," Journal of Zhejiang University Science B, vol. 12, no. 10, pp. 835-845, 2011.

[21] X. Zhang, J. A. Winkles, M. C. Gongora et al., “TWEAK-Fn14 pathway inhibition protects the integrity of the neurovascular unit during cerebral ischemia," Journal of Cerebral Blood Flow and Metabolism, vol. 27, no. 3, pp. 534-544, 2007.

[22] T. Yamashita, T. Kamiya, K. Deguchi et al., "Dissociation and protection of the neurovascular unit after thrombolysis and reperfusion in ischemic rat brain," Journal of Cerebral Blood Flow and Metabolism, vol. 29, no. 4, pp. 715-725, 2009.
[23] K. Hayakawa, T. Nakano, K. Irie et al., "Inhibition of reactive astrocytes with fluorocitrate retards neurovascular remodeling and recovery after focal cerebral ischemia in mice," Journal of Cerebral Blood Flow and Metabolism, vol. 30, no. 4, pp. 871-882, 2010.

[24] A. Arvidsson, T. Collin, D. Kirik, Z. Kokaia, and O. Lindvall, "Neuronal replacement from endogenous precursors in the adult brain after stroke," Nature Medicine, vol. 8, no. 9, pp. 963970, 2002.

[25] M. Gao, R. Liu, S. Y. Zhu, and G. H. Du, "Acute neurovascular unit protective action of pinocembrin against permanent cerebral ischemia in rats," Journal of Asian Natural Products Research, vol. 10, no. 6, pp. 551-558, 2008.

[26] Y. Qin, J. B. Wang, W. J. Kong et al., "The diarrhoeogenic and antidiarrhoeal bidirectional effects of rhubarb and its potential mechanism," Journal of Ethnopharmacology, vol. 133, no. 3, pp. 1096-1102, 2011.

[27] J. B. Wang, H. P. Zhao, Y. L. Zhao et al., "Hepatotoxicity or hepatoprotection? Pattern recognition for the paradoxical effect of the Chinese herb Rheum palmatum L. in treating rat liver injury," PLoS One, vol. 6, no. 9, Article ID e24498, 2011.

[28] E. Z. Longa, P. R. Weinstein, S. Carlson, and R. Cummins, "Reversible middle cerebral artery occlusion without craniectomy in rats," Stroke, vol. 20, no. 1, pp. 84-91, 1989.

[29] J. Chen, P. R. Sanberg, Y. Li et al., "Intravenous administration of human umbilical cord blood reduces behavioral deficits after stroke in rats," Stroke, vol. 32, no. 11, pp. 2682-2688, 2001.

[30] X. D. Chao, F. Fei, and Z. Fei, “The role of excitatory amino acid transporters in cerebral ischemia," Neurochemical Research, vol. 35, no. 8, pp. 1224-1230, 2010.

[31] Y. Liu, S. Mituska, K. Hashizume, T. Hosaka, and H. Nukui, "The time course of glucose metabolism in rat cerebral ischemia with middle cerebral artery occlusion-reperfusion model and the effect of MK-801," Neurological Research, vol. 18, no. 6, pp. 505-508, 1996.

[32] T. Nelson, E. E. Kaufman, and L. Sokoloff, "2-Deoxyglucose incorporation into rat brain glycogen during measurement of local cerebral glucose utilization by the 2-deoxyglucose method," Journal of Neurochemistry, vol. 43, no. 4, pp. 949-956, 1984.

[33] Y. Liu, S. Mituska, K. Hashizume, T. Hosaka, and H. Nukui, "The sequential change of local cerebral blood flow and local cerebral glucose metabolism after focal cerebral ischaemia and reperfusion in rat and the effect of MK-801 on local cerebral glucose metabolism," Acta Neurochirurgica, vol. 139, no. 8, pp. 770-779, 1997.

[34] M. He, Z. M. Xue, J. Li, and B. Q. Zhou, "Breviscapine inhibits high glucose-induced proliferation and migration of cultured vascular smooth muscle cells of rats via suppressing the ERK1/2 MAPK signaling pathway," Acta Pharmacologica Sinica, vol. 33, no. 5, pp. 606-614, 2012.

[35] Y. Liu, S. Mituska, K. Hashizume, T. Hosaka, and H. Nukui, "The time course of glucose metabolism in rat cerebral ischemia with middle cerebral artery occlusion-reperfusion model and the effect of MK-801," Neurological Research, vol. 18, no. 6, pp. $505-508,1996$.

[36] M. Lou, H. Zhang, J. Wang et al., "Hyperbaric oxygen treatment attenuated the decrease in regional glucose metabolism of rats subjected to focal cerebral ischemia: a high resolution positron emission tomography study," Neuroscience, vol. 146, no. 2, pp. 555-561, 2007. 
[37] W. Xu, R. P. Zha, W. Y. Wang, and Y. P. Wang, "Effects of scutellarin on PKC $\gamma$ in PC12 cell injury induced by oxygen and glucose deprivation," Acta Pharmacologica Sinica, vol. 28, no. 10, pp. 1573-1579, 2007.

[38] L. X. Wang, J. P. Zeng, X. B. Wei, F. W. Wang, Z. P. Liu, and X. M. Zhang, "Effects of scutellarin on apoptosis induced by cobalt chloride in PC12 cells," The Chinese Journal of Physiology, vol. 50, no. 6, pp. 301-307, 2007.

[39] H. Hong and G. Q. Liu, "Protection against hydrogen peroxideinduced cytotoxicity in PC12 cells by scutellarin," Life Sciences, vol. 74, no. 24, pp. 2959-2973, 2004.

[40] H. Liu, X. L. Yang, Y. Wang, X. Q. Tang, D. Y. Jiang, and H. B. Xu, "Protective effects of scutellarin on superoxideinduced oxidative stress in rat cortical synaptosomes," Acta Pharmacologica Sinica, vol. 24, no. 11, pp. 1113-1174, 2003.

[41] H. F. Zhang, X. M. Hu, L. X. Wang, S. Q. Xu, and F. D. Zeng, "Protective effects of scutellarin against cerebral ischemia in rats: evidence for inhibition of the apoptosis-inducing factor pathway," Planta Medica, vol. 75, no. 2, pp. 121-126, 2009.

[42] L. L. Lin, A. J. Liu, J. G. Liu, X. H. Yu, L. P. Qin, and D. F. Su, "Protective effects of scutellarin and breviscapine on brain and heart ischemia in rats," Journal of Cardiovascular Pharmacology, vol. 50, no. 3, pp. 327-332, 2007.

[43] J. Mykkänen, M. Juhola, and U. Ruotsalainen, "Threedimensional ROIs in brain PET," Studies in Health Technology and Informatics, vol. 68, pp. 339-342, 1999.

[44] E. Planel, T. Miyasaka, T. Launey et al., "Alterations in glucose metabolism induce hypothermia leading to tau hyperphosphorylation through differential inhibition of kinase and phosphatase activities: implications for Alzheimer's disease," Journal of Neuroscience, vol. 24, no. 10, pp. 2401-2411, 2004.

[45] H. Zhang, X. Zheng, X. Yang et al., "11C-NMSP/18F-FDG microPET to monitor neural stem cell transplantation in a rat model of traumatic brain injury," European Journal of Nuclear Medicine and Molecular Imaging, vol. 35, no. 9, pp. 1699-1708, 2008.

[46] S. Rex, P. T. Meyer, J. H. Baumert et al., "Positron emission tomography study of regional cerebral blood flow and flowmetabolism coupling during general anaesthesia with xenon in humans," British Journal of Anaesthesia, vol. 100, no. 5, pp. 667675, 2008.

[47] A. K. Håberg, H. Qu, and U. Sonnewald, "Acute changes in intermediary metabolism in cerebellum and contralateral hemisphere following middle cerebral artery occlusion in rat," Journal of Neurochemistry, vol. 109, supplement 1, pp. 174-181, 2009.

[48] S. J. Chmura, M. E. Dolan, A. Cha, H. J. Mauceri, D. W. Kufe, and R. R. Weichselbaum, "In vitro and in vivo activity of protein kinase $\mathrm{C}$ inhibitor chelerythrine chloride induces tumor cell toxicity and growth delay in vivo," Clinical Cancer Research, vol. 6, no. 2, pp. 737-742, 2000.

[49] Y. Liu, M. V. Cohen, and J. M. Downey, "Chelerythrine, a highly selective protein kinase $\mathrm{C}$ inhibitor, blocks the antiinfarct effect of ischemic preconditioning in rabbit hearts," Cardiovascular Drugs and Therapy, vol. 8, no. 6, pp. 881-882, 1994.

[50] N. Saito and Y. Shirai, "Protein kinase C $\gamma(\mathrm{PKC} \gamma)$ : function of neuron specific isotype," Journal of Biochemistry, vol. 132, no. 5, pp. 683-687, 2002.

[51] U. Ito, Y. Hakamata, E. Kawakami, and K. Oyanagi, "Temporary focal cerebral ischemia results in swollen astrocytic end-feet that compress microvessels and lead to focal cortical infarction,"
Journal of Cerebral Blood Flow and Metabolism, vol. 31, no. 1, pp. 328-338, 2011.

[52] M. Michaelides, J. Pascau, J. D. Gispert et al., "Dopamine D4 receptors modulate brain metabolic activity in the prefrontal cortex and cerebellum at rest and in response to methylphenidate," European Journal of Neuroscience, vol. 32, no. 4, pp. 668-676, 2010.

[53] G. Barreto, R. E. White, Y. Ouyang, L. Xu, and R. G. Giffard, "Astrocytes: targets for neuroprotection in stroke," Central Nervous System Agents in Medicinal Chemistry, vol. 11, no. 2, pp. 164-173, 2011.

[54] M. Pekny, C. Eliasson, R. Siushansian et al., "The impact of genetic removal of GFAP and/or vimentin on glutamine levels and transport of glucose and ascorbate in astrocytes," Neurochemical Research, vol. 24, no. 11, pp. 1357-1362, 1999.

[55] T. Mabuchi, J. Lucero, A. Feng, J. A. Koziol, and G. J. Del Zoppo, "Focal cerebral ischemia preferentially affects neurons distant from their neighboring microvessels," Journal of Cerebral Blood Flow and Metabolism, vol. 25, no. 2, pp. 257-266, 2005. 


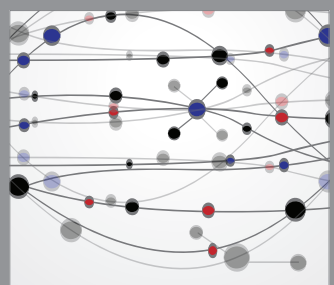

The Scientific World Journal
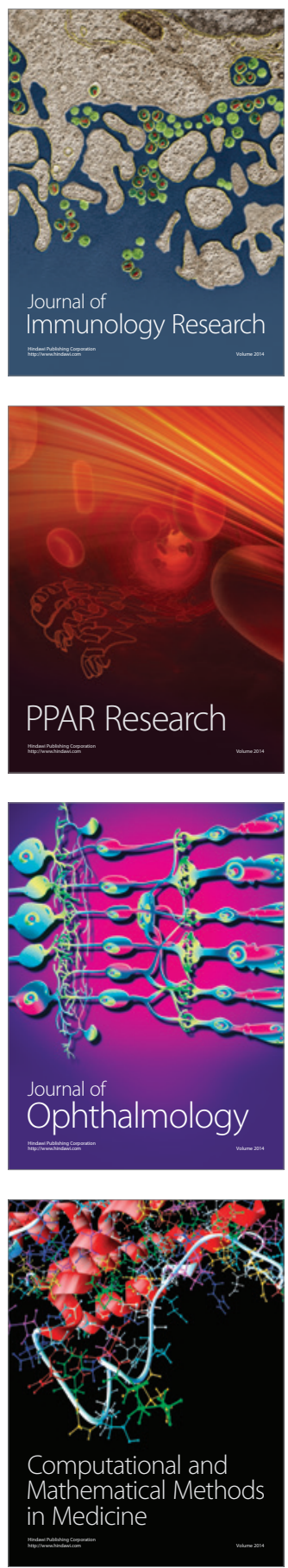

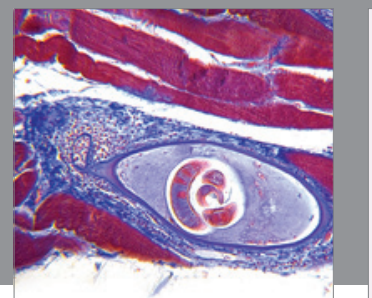

Gastroenterology

Research and Practice
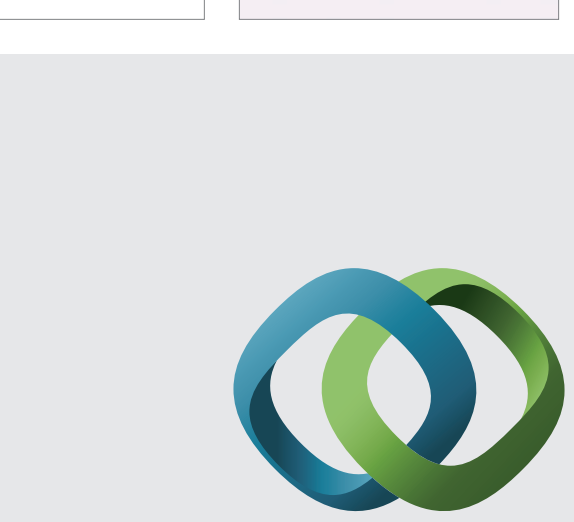

\section{Hindawi}

Submit your manuscripts at

http://www.hindawi.com
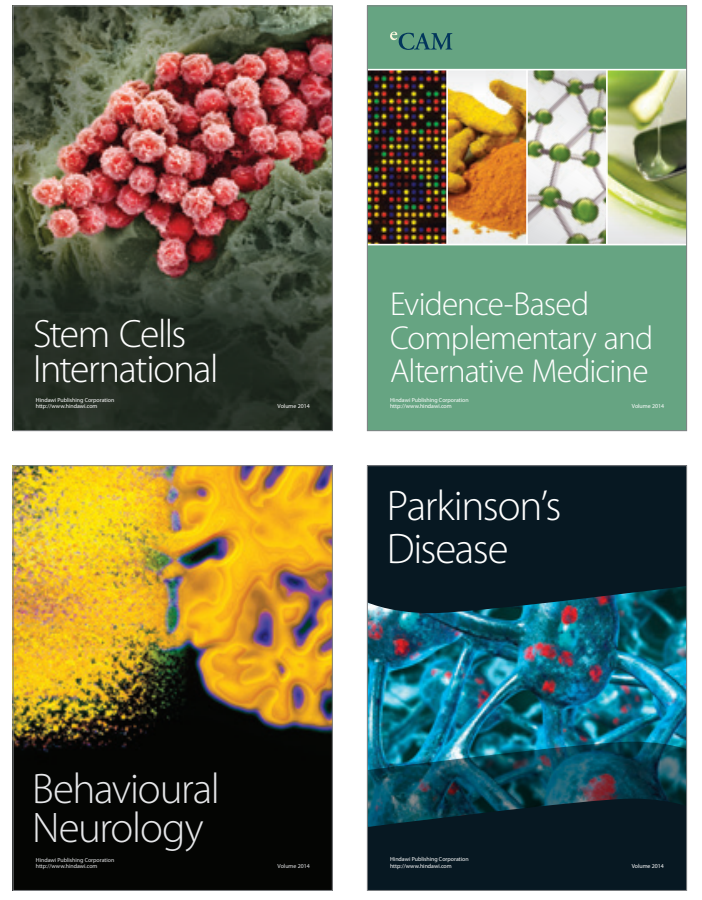
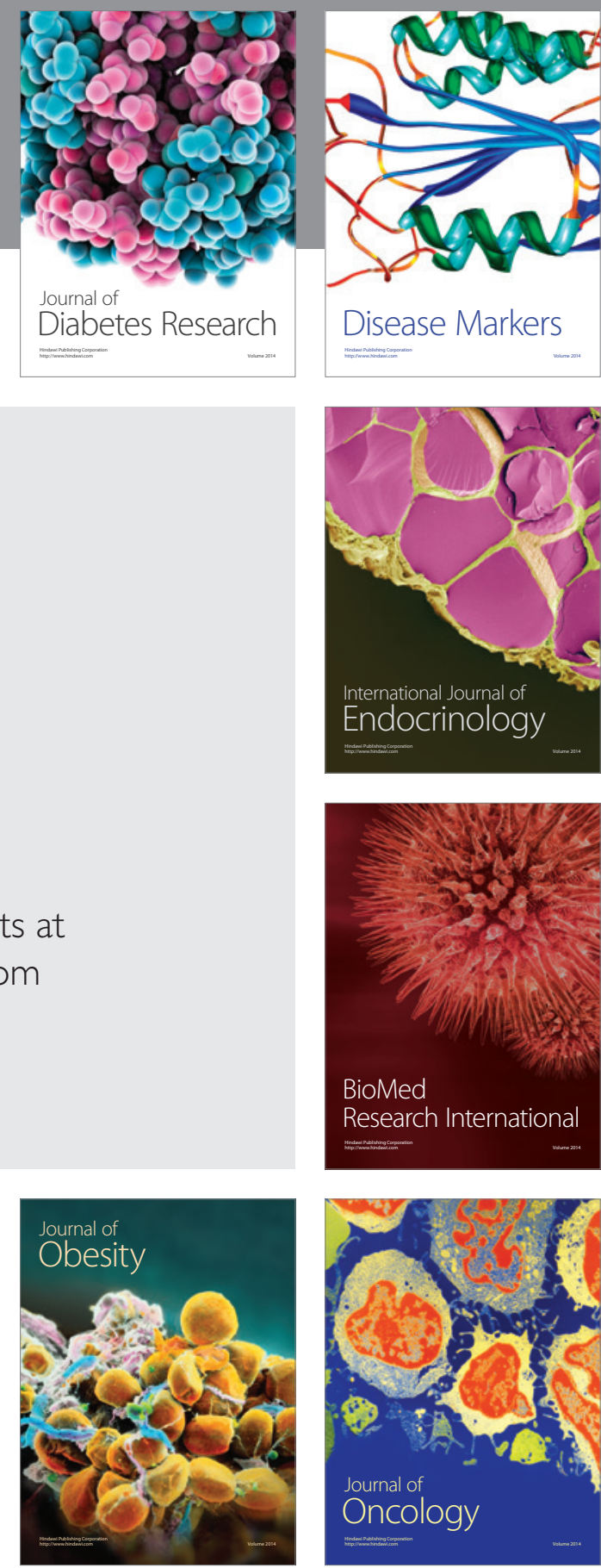

Disease Markers
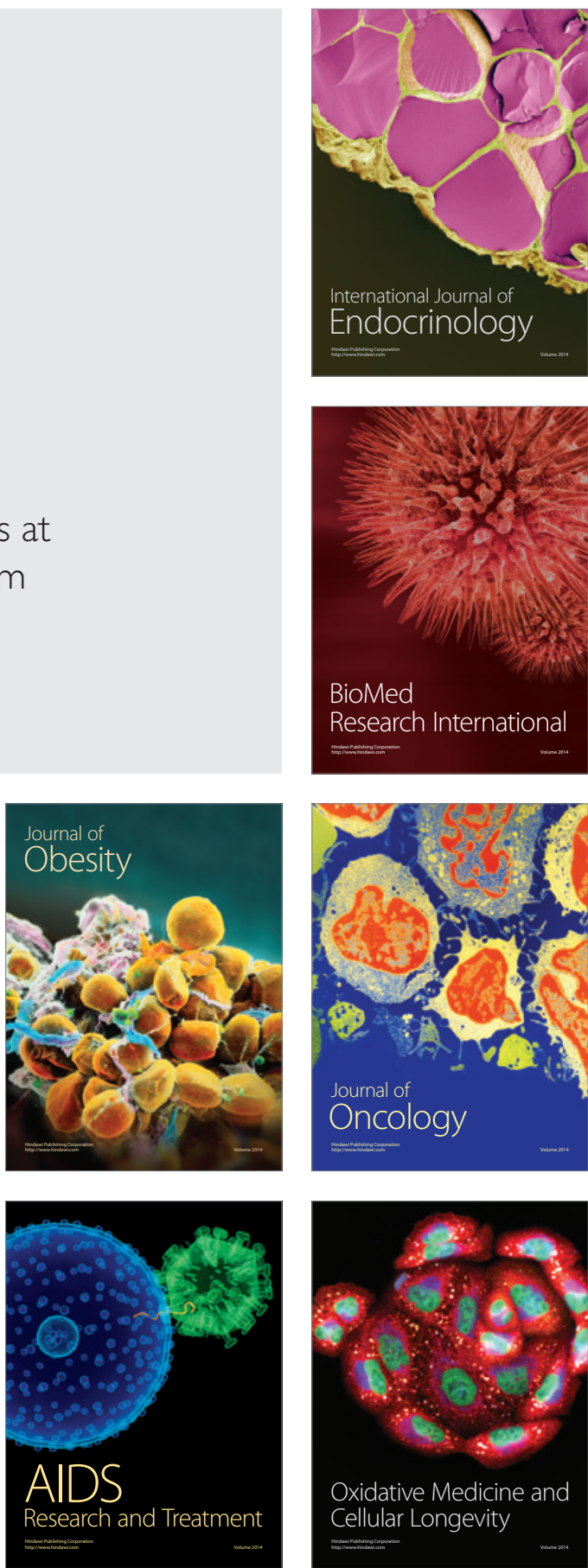